Conclusion: Medical laboratory technologists and students in central and southern Taiwan have a deficiency of knowledge about HIV/AIDS that may impact their attitudes. The medical laboratory profession should seek to change unfounded fears of HIV/AIDS for the betterment of the profession, their immediate families, and for patients with HIV/AIDS.

Keywords: acquired immune deficiency syndrome (AIDS); education; Human Immunodeficiency Virus (HIV); knowledge; medical laboratory technologists; Taiwan

prebosp Disast Med 2005;20(2):s55-556

Infectious Disease Preparedness after SARS-A Singapore Emergency Department's Experience L.S. Quek

National University Hospital, Singapore

It has been more than a year since severe acute respiratory syndrome (SARS) took the medical world by storm. Many lessons were learned from the outbreak, which changed the way many emergency departments manage potential infectious disease outbreaks. This poster highlights the changes and measures taken by our emergency department for future responses to such threats.

The following topics will be addressed: (1) resources for appropriate infection control system available for bioterrorism and emerging infectious diseases; (2) geographical isolation; (3) air handling and filtration system in the department; (4) command and control; (5) risk communication between patients, relatives, nursing, and medical staff; (6) patient, visitor and public information management; and (7) technology—radio frequency identification (RFID), and its application in contact tracing for patients and visitors coming to the department.

Keywords: emergency department; outbreak; preparedness; response; severe acute respiratory syndrome (SARS) Prebosp Disast Med 2005;20(2):s56

\section{The Global Epidemic Recognition and Management (GERM) Chart: A Prototype \\ P. Rega; ${ }^{1}$ K. Burkbolder-Allen, RN, MSEd; ${ }^{2}$ \\ C. Budd, RN, NREMT-P,2 M. Bisesi, PbD \\ 1. Medical College of Ohio, USA \\ 2. USA}

In the past three decades, North America has witnessed an increasing vulnerability to emerging infectious diseases. This vulnerability has been accentuated in recent years as unique threats such as Anthrax, severe acute respiratory syndrome (SARS), Norovirus, and Monkeypox have emerged. Despite a highly sophisticated healthcare environment, the public health aspects of an infectious disease epidemic remain problematic.

Old and new concepts such as quarantine, isolation, alternative care sites, surge capacity, prophylaxis, and pharmaceutical stockpiling are challenging to local experts and planners. In relation, the concepts are often difficult for infrastructure personnel, healthcare providers, and the average citizen to assimilate. To meet these challenges, the authors have developed a guidance tool for the implemen- tation of community-based planning in response to an outbreak and potential epidemic.

This guidance tool is divided into three categories outlining action plans for specific segments of the local infrastructure: Category 1-Healthcare Workers; Category 2Healthcare Facilities; and Category 3-Public Health Officials. Within each category, action plans have been developed in progressive phases and are dependent upon the location of an outbreak from a global perspective and its perceived threat to communities locally, regionally and nationally: Phase 1-Limited/No Outbreak (North America Unaffected); Phase 2-Multinational Outbreak (North America Unaffected); Phase 3-North American Outbreak (Local Community Unaffected); and Phase 4North American Outbreak (Local Community Affected).

The tool's composition not only prioritizes activities, but also enhances educational endeavors. While the tool contains action plans specific to North American communities, its general concepts, and educational aspects may be exported and personalized for use elsewhere.

Keywords: action plans; global epidemic recognition and management (GERM); North America; prototype; threats; tools Prebosp Disast Med 2005;20(2):556

\section{Examination of Continuous Aspiration of Subglottic Secretions}

\section{N. Okada $;{ }^{1}$ T. Kawashima, ${ }^{1}$ N. Ishii; ${ }^{1}$ A. Takahasbi ${ }^{1}$}

M. Okasbiro, ${ }^{1}$ T. Yosbida, ${ }^{1}$ K. Okuno ${ }^{2}$

1. Kobe University Hospital, Kobe, Japan

2. Ohara Hospital, Japan

Objective: Continuous aspiration of subglottic secretions (CASS) lately has been noted because of its effectiveness for preventing ventilator-associated pneumonia (VAP). However, the method used for aspiration has not been examined in detail, so the amount of secretion obtained with CASS was determined under several conditions.

Methods: This study was conducted at the High Care Unit (HCU) of the Kobe University and Ohara Hospitals. A total of 21 patients needing prolonged intubation with tracheotomy were studied. Three types of tracheotomy tubes were examined (Types A, B, and C). First, the amount of aspiration with CASS using the Type $A$ tube was determined during 30 days with regard to gender, size of the tracheotomy tube, respiratory condition (requiring ventilation or not), and swallowing function. Second, the amount of CASS with Type B or C tubes was determined during seven days. Continuous subglottic drainage was performed with around $45 \mathrm{mmHg}$.

Results: The average daily aspiration of the Type A tube was $47.8 \pm 50.3 \mathrm{ml}$, the most being $215.2 \pm 57.4 \mathrm{ml}$ and the least being $9.8 \pm 8.9 \mathrm{ml}$. Male patients, who were intubated using a large-sized tracheotomy tube, required mechanical ventilation, and had swallowing function, tended to have greater quantity with CASS, though no significant difference was shown. The average daily aspiration quantity of the Type B tube was $2.3 \pm 3.1 \mathrm{ml}$, and that of the Type C tube was $10.0 \pm 11.7 \mathrm{ml}$. Statistically significant differences were demonstrated between the 3 types of tracheotomy tubes. 